\title{
Válvula de Ahmed en glaucoma refractario. Comportamiento de la presión intraocular a 24 meses
}

\section{Ahmed valve in refractory glaucoma. Twenty-four-month intraocular pressure follow up}

\author{
Rodolfo Monti ${ }^{1}$, Evangelina Espósito ${ }^{1}$, María E. Forniés-Paz ${ }^{1}$, Nicolás Crim ${ }^{1}$, Juan Dalmagro , \\ Horacio M. Serra ${ }^{2}$, Julio A. Urrets-Zavalía ${ }^{1 *}$
}

${ }^{1}$ Servicio de Oftalmología, Clínica Universitaria Reina Fabiola, Universidad Católica de Córdoba; ${ }^{2} \mathrm{CIBICI}$-CONICET, Facultad de Ciencias Químicas, Universidad Nacional de Córdoba. Argentina

\section{Resumen}

Objetivo: Evaluar los resultados del implante de válvula de Ahmed en pacientes con diferentes tipos de glaucoma refractario. Pacientes, material y métodos: 14 ojos de 13 pacientes mayores de 18 años con glaucoma refractario al tratamiento médico máximo y operados mediante la implantación de una válvula de Ahmed (Modelo AGV-FP7) entre agosto de 2007 y abril de 2015, con un seguimiento mínimo de 24 meses, fueron evaluados retrospectivamente. Se consideró éxito absoluto cuando la presión intraocular (PIO) postoperatoria se hallaba entre 5 y $22 \mathrm{mmHg}$ sin tratamiento médico antiglaucomatoso; éxito relativo cuando la PIO postoperatoria se hallaba dentro de ese rango, pero con la ayuda de uno o más colirios antiglaucomatosos; y fracaso cuando la PIO postoperatoria era menor de 5 o mayor de $22 \mathrm{mmHg}$, o cuando se debió proceder al explante de la válvula. Resultados: En los pacientes con glaucoma crónico de ángulo abierto, el 14.29\% $(n=1)$ presentó éxito absoluto, el $57.14 \%(n=4)$, éxito relativo, y en el $28.57 \%(n=2)$ la cirugía valvular fracasó. En todos los pacientes con glaucoma pseudoexfoliativo, glaucoma asociado a uveítis y en glaucoma congénito se obtuvo un éxito relativo. Conclusiones: El implante valvular de Ahmed fue eficaz para reducir la PIO en glaucomas refractarios al tratamiento médico en un número importante de casos en esta serie, y se llegó a un éxito relativo en la mayoría de ellos.

Pallabras clave: Glaucoma refractario. Glaucoma complicado. Glaucoma secundario. Válvula de Ahmed. Cirugía de glaucoma. Glaucoma primario de ángulo abierto.

\section{Abstract}

Purpose: To evaluate the results of Ahmed valve implantation in refractory glaucoma patients. Patients, Material and Methods: 14 eyes in 13 patients with refractory glaucoma to medical treatment, which underwent an implantation of an Ahmed valve between August 2007 and April 2015, with a minimum follow up of 24 months were retrospectively reviewed. Success was defined as a postoperative intraocular pressure (IOP) between 5 and $22 \mathrm{mmHg}$ without antiglaucomatous medications, relative success when postoperative IOP was within that range but with one or more glaucoma drops, and failure when postoperative IOP was $<5$ or $>22 \mathrm{mmHg}$ or when the valve was explanted. Results: An absolute success was obtained in $14.29 \%(n=1)$ of patients with primary open angle glaucoma a relative success in $57.14 \%(n=4)$, and a

Correspondencia:

*Julio A. Urrets-Zavalía

E-mail: julioaurrets@gmail.com

DOl: $1024875 / \mathrm{RMO} M 18000019$
Disponible en internet: 06-04-2018 Rev Mex Oftalmol. 2018;92(2):94-100 www.rmo.com.mx

0187-4519/○ 2017 Sociedad Mexicana de Oftalmología. Publicado por Permanyer México. Este es un artículo Open Access bajo la licencia CC BY-NC-ND (http://creativecommons.org/licenses/by-nc-nd/4.0/). 
failure in $28.57 \%(n=2)$. Patients with pseudoexfoliative glaucoma, uveitic glaucoma and congenital glaucoma, showed $100 \%$ relative success. Conclusions: Ahmed valve implantation was effective in reducing IOP in glaucoma refractory to medical treatment in a significant number of cases in this series, reaching a relative success in most of them.

Key words: Refractory glaucoma. Complicated glaucoma. Secondary glaucoma. Ahmed valve. Claucoma surgery. Primary open angle glaucoma.

\section{Introducción}

Se denomina glaucoma refractario a cualquier tipo de glaucoma que no haya respondido al tratamiento médico máximo después del fracaso de una o más intervenciones quirúrgicas por glaucoma ${ }^{1}$. El manejo del glaucoma refractario es una tarea difícil para cualquier cirujano de glaucoma. Diversos dispositivos de drenaje han sido utilizados en el tratamiento del glaucoma refractario en adultos y niños, especialmente cuando la cirugía filtrante ha fallado o se sabe que tiene un alto riesgo de fracaso ${ }^{2-6}$.

La válvula de Ahmed, como su nombre indica, es un dispositivo valvulado de drenaje del humor acuoso, compuesto por un reservorio siliconado de forma elíptica que se implanta bajo la conjuntiva en la zona ecuatorial del globo ocular, y un tubo de silicona cuyo extremo anterior se aloja en la cámara anterior. Existen diversos modelos que varían en tamaño y diseño. Su objetivo es la reducción de la presión intraocular (PIO) mediante la salida del humor acuoso a través del tubo de conexión y su reabsorción en la ampolla de filtración que se forma alrededor del reservorio ${ }^{7}$.

El objetivo de este estudio es el de evaluar los resultados del implante de una válvula de Ahmed en pacientes con glaucoma refractario.

\section{Pacientes, material y métodos}

Fueron evaluados retrospectivamente 14 ojos de 13 pacientes con glaucoma refractario al tratamiento médico máximo que se sometieron a la implantación de una válvula de Ahmed de forma consecutiva entre agosto de 2007 y abril de 2015, con un seguimiento mínimo de 24 meses (Tabla 1). Todas las cirugías fueron realizadas por el mismo cirujano (JAD). Se utilizó una válvula de Ahmed modelo AGV-FP7 (New World Medical, Rancho Cucamonga, CA, EE.UU.), compuesta, por una parte, por un cuerpo o plato siliconado de forma elíptica de $13 \mathrm{~mm}$ de ancho por $16 \mathrm{~mm}$ de largo y una superficie total de $184 \mathrm{~mm}^{2}$, que se implanta bajo la conjuntiva en la zona ecuatorial del globo ocular y en cuyo interior se aloja una cámara trapezoidal que genera un efecto Venturi que facilita la circulación del humor acuoso, y, por otra parte, por un tubo de silicona de $25 \mathrm{~mm}$ de longitud, con un diámetro externo de $0.63 \mathrm{~mm}$ e interno de $0.30 \mathrm{~mm}$.

Los datos evaluados fueron la edad en el momento de la cirugía, la forma clínica de glaucoma, la PIO media pre y postoperatoria, el seguimiento promedio en meses, la necesidad de medicación tópica para glaucoma después de la cirugía y la presentación de complicaciones después de la cirugía. La PIO fue evaluada en todos los pacientes con tonómetro de aplanación de Goldmann. La PIO postoperatoria fue evaluada a la primera semana, al mes, y a los 6, 12 y 24 meses.

\section{Técnica quirúrgica}

Después de la preparación del campo quirúrgico con iodopovidona al 10\%, la realización de la anestesia peribulbar con lidocaína $2 \%$ y la fijación del globo ocular mediante la tracción de los rectos, o bien mediante puntos de tracción corneales, se realiza un colgajo conjuntival de base en fórnix. Se diseca ampliamente la conjuntiva y la cápsula de Tenon para exponer el cuadrante temporal superior donde luego será suturado el reservorio. Se coloca el reservorio entre los músculos y por debajo de la cápsula de Tenon, rotándolo posteriormente en el plano horizontal para colocarlo correctamente con el tubo de conexión orientado hacia la parte anterior del globo ocular. El reservorio se sutura a la esclera a unos 8-10 mm del limbo, utilizando suturas no reabsorbibles de nailon $5 / 0$ a $8 / 0$. El túnel de entrada para el tubo se realiza a $4 \mathrm{~mm}$ del limbo esclerocorneal, mediante punción de la esclera con una aguja calibre $23 \mathrm{G}$, luego se introduce el tubo en la cámara anterior, situándolo en un plano paralelo al iris, a media distancia entre el iris y la córnea, siempre evitando el roce del tubo con el endotelio corneal. Por último, se cierra la conjuntiva con suturas reabsorbibles de Vicryl 8/0. Finalizada la cirugía, se coloca en el fondo de saco conjuntival inferior una pomada con dexametasona y un antibiótico de amplio espectro y se ocluye el ojo durante 24 horas.

Se consideró éxito absoluto de la cirugía cuando la $\mathrm{PIO}$ postoperatoria se hallaba entre 5 y $22 \mathrm{mmHg} \sin$ 


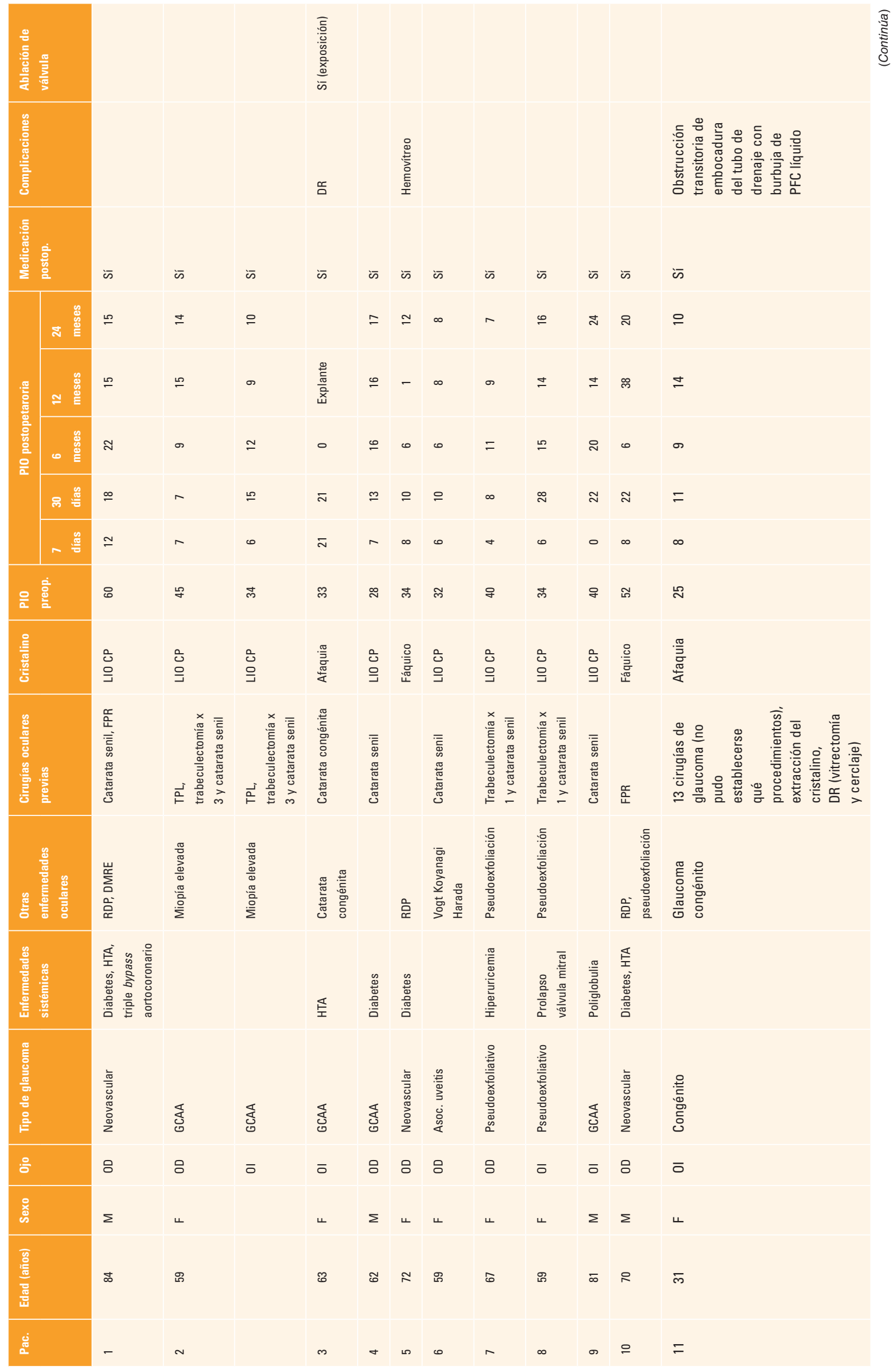




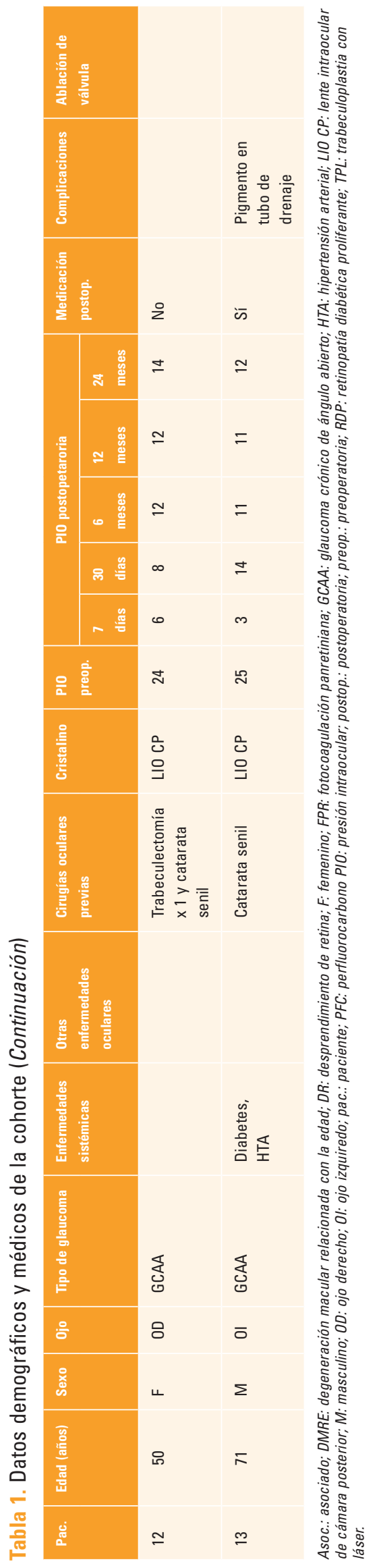

tratamiento médico antiglaucomatoso; éxito relativo cuando la PIO postoperatoria se hallaba dentro de ese rango, pero con la ayuda de uno o más colirios antiglaucomatosos; y fracaso cuando la PIO postoperatoria era menor de 5 o mayor de $22 \mathrm{mmHg}$, o cuando se debió proceder al explante de la válvula.

El presente estudio fue aprobado por el Comité Institucional de Ética de la Investigación en Salud de nuestra institución.

\section{Método estadístico}

Se utilizó estadística descriptiva y de frecuencias para las variables de edad, sexo y tipo de glaucoma. Para la estadística analítica, las mediciones de PIO preoperatoria y postoperatoria se compararon mediante la prueba de Friedman. El análisis de normalidad de las variables fue realizado mediante el Test de Shapiro-Wilks. Se utilizó el Wilcoxon signed rank test para comparar los cambios en el número de medicamentos oculares. Se consideró significancia estadística cuando $p<0.05$.

\section{Resultados}

La edad media de los pacientes fue de $63 \pm 13$ años, rango $=31-84$ años. El $62 \%$ de los pacientes fueron mujeres. Las formas clínicas de glaucoma fueron: 6 (7 ojos) pacientes con glaucoma crónico de ángulo abierto (GCAA) (50\%), 3 (3 ojos) con glaucoma neovascular (21.4\%), 2 (2 ojos) con glaucoma pseudoexfoliativo (14.3\%), 1 (1 ojo) con glaucoma asociado a uveítis (7.1\%) y 1 (1 ojo) con glaucoma congénito (ya operado en múltiples ocasiones) (7.1\%) (Tabla 1). La PIO media preoperatoria fue de $36.14 \pm 10.49$, rango $=24$ $60 \mathrm{mmHg}$. Después de la cirugía se observó un descenso importante y sostenido de la PIO a lo largo de los 24 meses de seguimiento mínimo (Fig. 1). La PIO presentó una distribución no normal (Test de Shapiro-Wilks). La PIO media postoperatoria a la primera semana fue de $7.29 \pm 4.81$, rango $=0-21 ; p<0.0001$; al mes, $14.79 \pm 6.47$, rango $=7-28 ; p=0.0087$; a los 6 meses, $11.07 \pm 5.88$, rango $=0-22 ; p<0.0001$; a los 12 meses, $13.54 \pm 8.40$, rango $=1-38 ; p=0.0002 ; y$ a los 24 meses, $13.77 \pm 4.78 \mathrm{mmHg}$, rango = 7-24; $\mathrm{p}=0.0043$ (Fig. 2 y Tabla 2). El seguimiento promedio fue 30 meses, rango = 24-54 meses. $\mathrm{A}$ los 24 meses de la cirugía, el $7.14 \%$ de los pacientes presentaron un éxito absoluto, el $78.57 \%$, un éxito relativo, y un fracaso del tratamiento de $14.29 \%$ (Tabla 2). Solo un paciente 


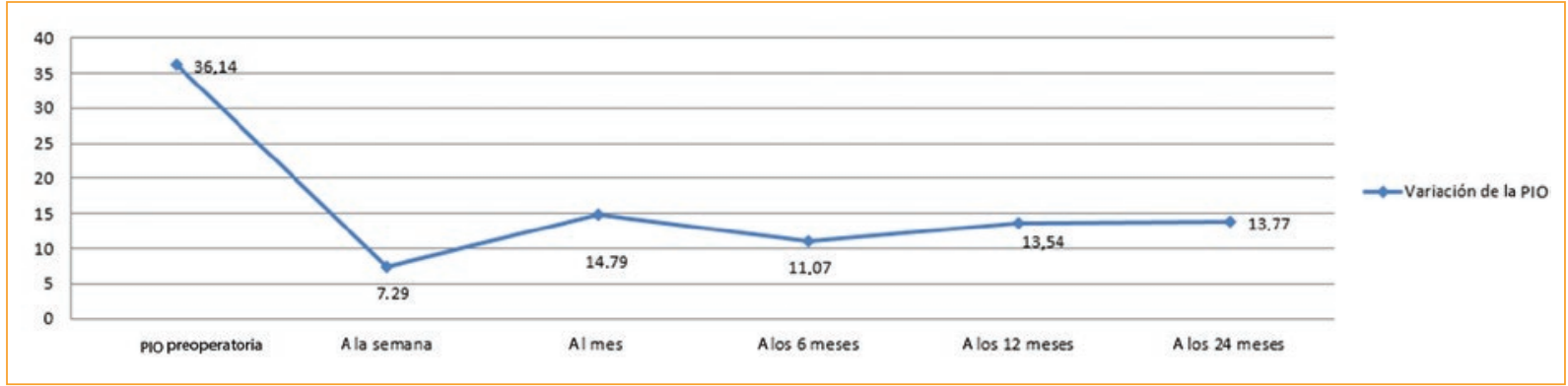

Figura 1. Gráfico en el que se observa la reducción postoperatoria de la PIO y su mantenimiento durante 24 meses de seguimiento mínimo. PIO: presión intraocular.

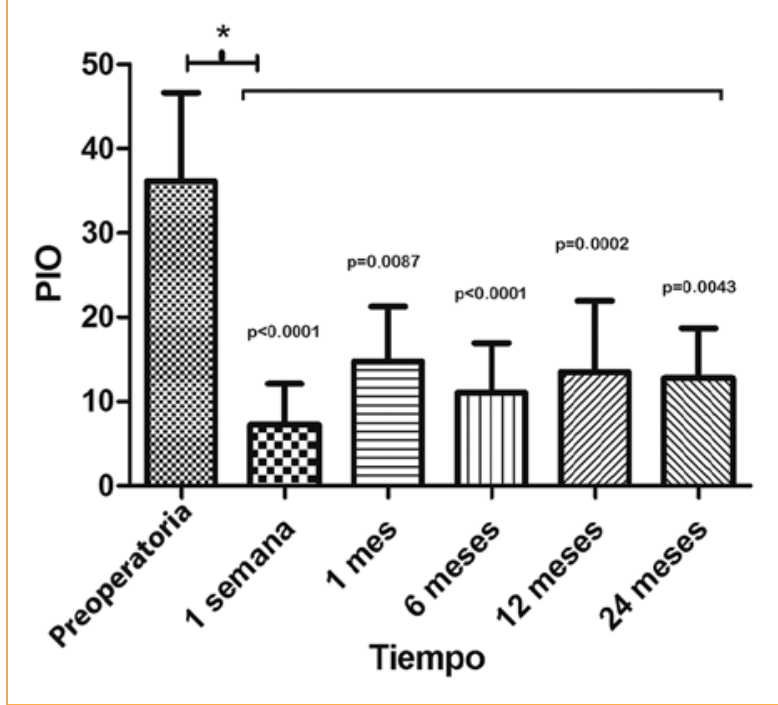

Figura 2. Gráfico en el que se puede observar comparativamente la variación de la PIO a lo largo de los 24 meses de seguimiento postoperatorio mínimo. PIO: presión intraocular.

requirió el explante de la válvula por exposición a los 12 meses postoperatorios.

Según la forma clínica preoperatoria de glaucoma, en el GCAA, el $14.29 \%$ presentó éxito absoluto, $57.14 \%$, éxito relativo, y $28.57 \%$, fracaso. En pacientes con glaucoma pseudoexfoliativo, glaucoma asociado a uveítis y glaucoma congénito, el $100 \%$ presentó un éxito relativo (Fig. 3). El promedio del número de medicaciones tópicas preoperatorias fue de $2.85 \pm 0.8$, rango $=3-5$, y a los 24 meses postoperatorios, de 2.64 \pm 0.93 , rango $=0-5 ; p=0.7451$.

En el periodo postquirúrgico, una fase hipertensiva fue registrada para el $70 \%$ de los casos. Fue necesario comenzar la medicación hipotensora a la segunda semana en un $39 \%(n=5)$ de los casos, seguida de la sexta semana en un $31 \%(n=4)$ de los casos.
Tabla 2. Resultados postoperatorios después de un implante valvular de Ahmed en pacientes con glaucoma refractario

\begin{tabular}{|c|c|}
\hline PIO media preoperatoria $(\mathrm{mmHg})$ & $36.14 \pm 10.49(r=24-60)$ \\
\hline PIO media postoperatoria $(\mathrm{mmHg})$ & $13.77 \pm 4.78(r=7-24)$ \\
\hline Seguimiento (meses) & $30(r=24-54)$ \\
\hline $\begin{array}{l}\text { Resultado a los } 24 \text { meses } \\
\text { Éxito absoluto } \\
\text { Éxito relativo } \\
\text { Fracaso }\end{array}$ & $\begin{array}{l}7.14 \% \\
78.57 \% \\
14.29 \%\end{array}$ \\
\hline $\begin{array}{l}\text { Éxito (relativo y absoluto) según } \\
\text { forma clínica } \\
\text { GCAA } \\
\text { Glaucoma neovascular } \\
\text { Glaucoma pseudoexfoliativo } \\
\text { Glaucoma asociado a uveítis } \\
\text { Glaucoma congénito }\end{array}$ & $\begin{array}{l}71.43 \% \\
100 \% \\
100 \% \\
100 \% \\
100 \%\end{array}$ \\
\hline $\begin{array}{l}\text { Promedio de medicación tópica } \\
\text { Preoperatoria } \\
\text { Postoperatoria }\end{array}$ & $\begin{array}{l}2.85 \pm 0.80(r=3-5) \\
2.64 \pm 0.93(r=0-5)\end{array}$ \\
\hline
\end{tabular}

GCAA: glaucoma crónico de ángulo abierto; PIO: presión intraocular.

En ninguno de los casos se empleó antimetabolitos locales como coadyuvantes. En el paciente 10, portador de un glaucoma neovascular secundario a retinopatía diabética proliferante ya fotocoagulada, se realizó inyección intravítrea de $1.25 \mathrm{mg} / 0.05 \mathrm{ml}$ de bevacizumab (Avastin ${ }^{\circledR}$, Roche Diagnostic GmbH, Manheim, Germany) 7 días antes de la cirugía valvular a fin de controlar la neovascularización aún activa del iris y el ángulo iridocorneal.

\section{Discusión}

En los últimos años se han desarrollado alternativas terapéuticas para los pacientes con glaucoma refractario al tratamiento convencional, como el tratamiento 


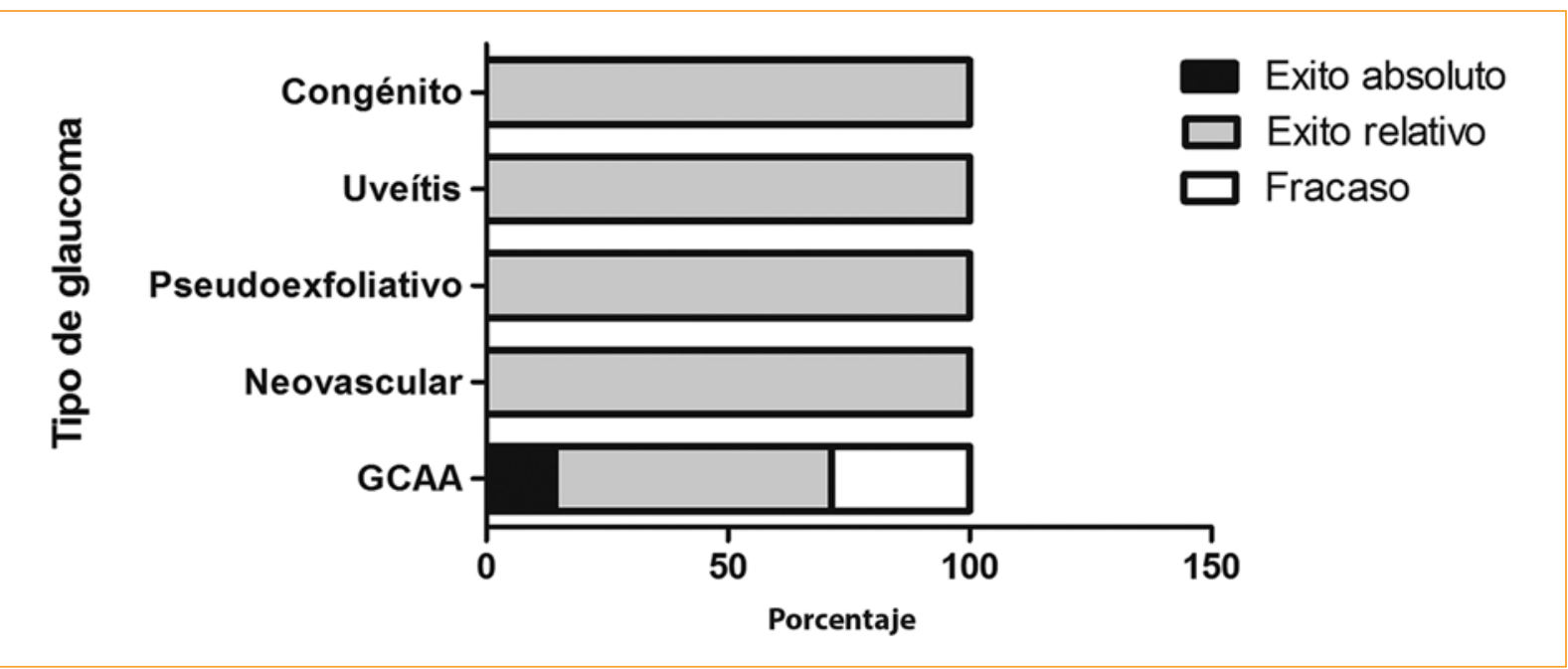

Figura 3. Gráfico en el que se observa el porcentaje de éxito en cada grupo según el tipo de glaucoma. GCAA: glaucoma crónico de ángulo abierto; PIO: presión intraocular.

con antimetabolitos en la trabeculectomía y diferentes procedimientos ciclodestructivos. Estas alternativas no logran controlar la $\mathrm{PIO}$ en todos los pacientes, y ponen al paciente en riesgo de desarrollar potencialmente graves complicaciones como ojo seco, úlceras de córnea, descompensación endotelial, hipema, iritis, bloqueo pupilar, desprendimiento coroideo e hipotonía ${ }^{8}$.

Los implantes valvulares para el glaucoma han crecido en popularidad en los últimos años, y eso se debe a una mejora en el tipo de implantes disponibles y a un mejor conocimiento acerca de su manejo. Actualmente contamos con implantes valvulados (Ahmed) y no valvulados (Molteno, Baerveldt). Los porcentajes de éxito reportados son del $90 \%$ en la mayoría de los glaucomas, incluso en los de mal pronóstico como son el neovascular y el uveítico ${ }^{8}$. Nuestro trabajo presenta los resultados que obtuvimos después del implante valvular de Ahmed en nuestros pacientes con glaucoma refractario. La válvula de Ahmed fue seleccionada debido a que evita la hipotonía grave postoperatoria y a que tiene un perfil más seguro ${ }^{9}$, aunque otros grupos reportan haber obtenido mejores resultados con el modelo Baerveldt ${ }^{10}$.

Los resultados de esta investigación demuestran que el dispositivo fue eficaz y seguro para disminuir la PIO en el tratamiento de glaucoma refractario, concordando con otros estudios que también hablan de una reducción significativa de la $\mathrm{PIO}^{5,7,11,12}$.En esta serie de pacientes solo se presentó una complicación postoperatoria, relacionada con un traumatismo ocular contuso que provocó la exposición del dispositivo de drenaje. Posiblemente, en el seguimiento a largo plazo de estos pacientes se podrían presentar otras complicaciones relacionadas a la válvula.

Según Yang y Park ${ }^{13}$, el éxito quirúrgico global fue aproximadamente del $81 \%$ en su heterogéneo grupo de pacientes, similar a los resultados de otros estudios de implantes, aunque el número de pacientes, criterios de éxito y longitud de seguimiento muestra una amplia variación ${ }^{13}$. Estas diferencias interestudio hacen difícil comparar con exactitud las proporciones de éxito entre los diversos reportes de implantes. Estas diferencias podrían resultar de la variedad de pacientes con diferentes patologías oculares, procedimientos quirúrgicos oftalmológicos previos al implante, diferentes edades en el momento de implantación, afaquia y circunstancias relacionadas a la técnica quirúrgica. La cirugía de implante valvular requiere que el cirujano esté familiarizado con la técnica quirúrgica y con el estrecho seguimiento postoperatorio a fin de evitar o controlar posibles complicaciones, como la fibrosis perivalvular, y así lograr el correcto funcionamiento de la válvula por un tiempo prolongado. Generalmente, las complicaciones posquirúrgicas tempranas descritas incluyen hipotonía, cámara anterior baja, hipema, aumento transitorio de la PIO y oclusión del tubo, mientras que las complicaciones a largo plazo incluyen encapsulamiento, alteración de la posición del tubo intracamerular, descompensación endotelial y endoftalmitis ${ }^{7,14}$.

En el estudio de Coleman, et al. ${ }^{11}$, en el que se evaluó prospectivamente la evolución clínica de 68 ojos sometidos a la colocación de válvula de Ahmed, se reportó una probabilidad acumulada de éxito a los 12 meses del $78 \%$, en concordancia con el porcentaje de éxito total a los 12 meses (78.57\%) observada en nuestra serie. Esto 
es comparable también al dispositivo iStent ${ }^{\circledR}$, que in vitro ha demostrado mejorar la facilidad del drenaje de humor acuoso en un $84 \%$ de los casos $^{12}$.

La presencia de una fase hipertensiva después de la cirugía es definida como una PIO superior a $21 \mathrm{mmHg}$ durante los 3 primeros meses posquirúrgicos. Los resultados permiten afirmar que en esta cohorte, después de la cirugía, hubo una etapa hipertensiva a la segunda y sexta semana, respectivamente, lo que coincide con lo descrito en la bibliografía. Esto es comparable a estudios previos que mostraron una fase hipertensiva en un 25 a $82 \%$ después del implante ${ }^{15,16}$.

En un estudio publicado por Parihar, et al., las formas clínicas encontradas fueron glaucoma juvenil de inicio tardío en 6 casos, glaucoma postraumático en 12 casos, glaucoma secundario posquirúrgico en 8 casos, glaucoma refractario de ángulo cerrado en 15 casos y glaucoma refractario de ángulo abierto en 11 casos, y se alcanzó un éxito total en 46 ojos $(88 \%)^{17}$. En nuestra serie, el éxito obtenido fue del $85.7 \%$ de los casos.

Si bien nuestra experiencia inicial en esta serie es alentadora en el largo plazo, la limitación del estudio radica en el limitado número de pacientes y en lo heterogéneo de las formas clínicas analizadas. Las publicaciones en idioma español indexadas sobre el tema son escasas, por lo que consideramos que nuestro trabajo podría ser de utilidad para la numerosa comunidad oftalmológica de habla hispana.

En conclusión, el implante valvular de Ahmed fue eficaz para reducir la $\mathrm{PIO}$ en glaucomas refractarios al tratamiento médico en un número importante de casos en esta serie, llegando a un éxito relativo en la mayoría de ellos.

\section{Responsabilidades éticas}

Protección de personas y animales. Los autores declaran que para esta investigación no se han realizado experimentos en seres humanos ni en animales.

Confidencialidad de los datos. Los autores declaran que han seguido los protocolos de su centro de trabajo sobre la publicación de datos de pacientes.

Derecho a la privacidad y consentimiento informado. Los autores han obtenido el consentimiento informado de los pacientes y/o sujetos referidos en el artículo. Este documento obra en poder del autor de correspondencia.

\section{Financiamiento}

Los autores declaran no poseer interés económico alguno en el/los productos mencionados en el presente trabajo, como tampoco haber recibido ayuda económica para la realización del mismo.

\section{Conflicto de intereses}

No existe ningún conflicto de interés de los investigadores en este estudio.

\section{Bibliografía}

1. Valtot F. Actualités sur les glaucomes réfractaires. J Fr Ophtalmol. 2003:26:56-61.

2. Topouzis F, Coleman AL, Choplin N, Bethlem MM, Hill R, Yu F, et al. Follow-up of the original cohort with the Ahmed glaucoma valve implant. Am J Ophthalmol. 1999;128:198-4.

3. Lai J, Poon A, Chua J, Tham C, Leung A, Lam D. Efficacy and safety of the Ahmed glaucoma valve implant in Chinese eyes with complicated glaucoma. Br J Ophthalmol. 2000;84:718-21.

4. Netland PA, Walton DS. Glaucoma drainage implants in pediatric patients. Ophthalmic Surg. 1993;24:723-9.

5. Colás-Tomás T, Gutiérrez-DíazE, Tejeda-PalaciosP,Barceló-Mendiguchía A, Mencía-Gutiérrez E. Resultados a medio plazo de dispositivos de drenaje para glaucoma en pacientes pediátricos. Arch Soc Esp Oftalmol. 2012;87:38-43.

6. Lee HY, Park JS, Choy YJ, Lee HJ. Surgical outcomes of different Ahmed Glaucoma Valve implantation methods between scleral graft and scleral flap. Korean J Ophthalmol. 2011;25:317-22.

7. Gutiérrez-Díaz E, Montero-Rodríguez M. Dispositivos de drenaje para glaucoma. Madrid: Ergon; 2002. p. 119-27.

8. Diaz-Llopis M, Salom D, García-Delpech S, Udaondo P, Millan JM, Arevalo JF. Efficacy and safety of the pars plana clip in the Ahmed valve device inserted via the pars plana in patients with refractory glaucoma. Clin Ophtalmol. 2010;4:411-16.

9. Budenz DL, Feuer WJ, Barton K, Schiffman J, Costa VP, Godfrey DG, et al. Postoperative complications in the Ahmed Baerveldt Comparison Study during five years of follow-up. Am J Ophthalmol. 2016;163:75-82.

10. Christakis PG, Kalenak JW, Tsai JC, Zurakowski D, Kammer JA, Harasymowycz PJ, et al. The Ahmed Versus Baerveldt Study: five-year treatment outcomes. Ophthalmology. 2016;123:2093-102.

11. Coleman AL, Hill R, Wilson MR, Choplin N, Kotas-Neumann R, Tam M, et al. Initial clinical experience with the Ahmed Glaucoma Valve Implant. Am J Ophthalmol. 1995;120:23-31.

12. Bahler CK, Smedley GT, Zhou J, Johnson DH. Trabecular bypass stents decrease intraocular pressure in cultured human anterior segments. Am J Ophthalmol. 2004;138:988-94.

13. Yang HK, Park KH. Clinical outcomes after Ahmed valve implantation in refractory paediatric glaucoma. Eye. 2009;23:1427-35.

14. Zarei R, Amini H, Daneshvar R, Nabi FN, Moghimi S, Fakhraee G, et al. Long-term Outcomes of Ahmed Glaucoma Valve Implantation in Refractory Glaucoma at Farabi Eye Hospital, Tehran, Iran. Middle East Afr J Ophthalmol. 2016;23:104-9.

15. Nouri-Mahdavi K, Caprioli J. Evaluation of the hypertensive phase after insertion of the Ahmed glaucoma valve. Am J Ophthalmol. 2003; 136:1001-8

16. Chen TC, Bhatia LS, Walton DS. Ahmed valve surgery for refractory pediatric glaucoma: a report of 52 eyes. J Pediatr Ophthalmol Strabismus. 2005;42:274-83.

17. Parihar JKS, Vats DP, Maggon R, Mathur V, Singh A, Mishra SK. The efficacy of Ahmed glaucoma valve drainage devices in cases of adult refractory glaucoma in Indian eyes. Indian J Ophthalmol. 2009;57:345-50. 